\title{
A giant pelvic solitary fibrous tumor with Doege-Potter syndrome successfully treated with transcatheter arterial embolization followed by surgical resection: a case report
}

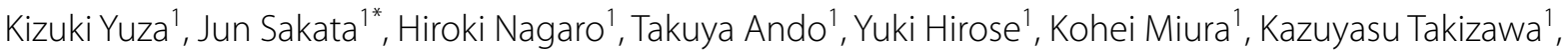 \\ Takashi Kobayashi' ${ }^{1}$ Hiroshi Ichikawa' ${ }^{1}$, Takaaki Hanyu' ${ }^{1}$, Yoshifumi Shimada' ${ }^{1}$ Masayuki Nagahashi', \\ Shin-Ichi Kosugi ${ }^{2}$ and Toshifumi Wakai ${ }^{1}$
}

\begin{abstract}
Background: Solitary fibrous tumor (SFT), a mesenchymal fibroblastic tumor with a hypervascular nature, rarely develops in the pelvis. Resection of a giant SFT occupying the pelvic cavity poses an increased risk of developing massive hemorrhage during resection, although surgical resection is the most effective treatment method for this tumor to achieve a potential cure. SFT rarely develops with Doege-Potter syndrome, which is known as a paraneoplastic syndrome characterized by non-islet cell tumor hypoglycemia (NICTH) secondary to SFT that secretes insulin-like growth factor-II (IGF-II). We present a case of a giant pelvic SFT with Doege-Potter syndrome, which was successfully treated with transcatheter arterial embolization (TAE) followed by surgical resection.

Case presentation: A 46-year-old woman presented with a disorder of consciousness due to refractory hypoglycemia. Images of the pelvis showed a giant and heterogeneously hypervascular mass displacing and compressing the rectum. Endocrinological evaluation revealed low serum levels of insulin and C-peptide consistent with NICTH. Angiography identified both the inferior mesenteric artery and the bilateral internal iliac artery as the main feeders of the tumor. To avoid intraoperative massive bleeding, super-selective TAE was performed for the tumor 2 days prior to surgery. Hypoglycemia disappeared after TAE. The tumor was resected completely, with no massive hemorrhage during resection. Histologically, it was diagnosed as IGF-II-secreting SFT. Partial necrosis of the rectum in the specimen was observed due to TAE. The patient was followed up for 2 years and no evidence of disease has been reported.

Conclusions: Preoperative angiography followed by TAE is an exceedingly helpful method to reduce intraoperative hemorrhage when planning to resect SFT occupying the pelvic cavity. Complications related to ischemia should be kept in mind after TAE, which needs to be planned within 1 or 2 days before surgery. TAE for tumors may be an option in addition to medical and surgical treatment for persistent hypoglycemia in Doege-Potter syndrome.
\end{abstract}

Keywords: Solitary fibrous tumor, Non-islet cell tumor hypoglycemia, Doege-Potter syndrome, Insulin-like growth factor-II, Preoperative transcatheter arterial embolization, Intestinal ischemia, Surgical resection

*Correspondence: jsakata2@med.niigata-u.ac.jp

${ }^{1}$ Division of Digestive and General Surgery, Niigata University Graduate School of Medical and Dental Sciences, 1-757 Asahimachi-dori, Chuo-ku, Niigata, Niigata 951-8510, Japan

Full list of author information is available at the end of the article

\section{Background}

Solitary fibrous tumor (SFT) is a rare mesenchymal fibroblastic tumor with a hypervascular nature, which most commonly develops in the pleura followed by the retroperitoneal and abdominopelvic cavity [1]. SFT that develops in the pelvis is sometimes discovered as a giant 
tumor, which poses an increased risk of developing massive hemorrhage during resection, although surgical resection is the most effective treatment method for SFTs to achieve a potential cure. This tumor rarely develops with non-islet cell tumor hypoglycemia (NICTH), which is known as a paraneoplastic syndrome caused by extrapancreatic tumors that secrete insulin-like growth factorII (IGF-II) [2]. This clinical condition caused by SFT is called Doege-Potter syndrome. In this case report, we present our experience of a giant pelvic SFT with DoegePotter syndrome, which was successfully treated with transcatheter arterial embolization (TAE) followed by surgical resection.

\section{Case presentation}

A 46-year-old woman was referred to our hospital for further examination and treatment of persistent hypoglycemia and a giant pelvic mass. She had no relevant medical history, including diabetes. No evident mass was palpable in the abdomen. Laboratory examination, including tumor markers, revealed no abnormalities except for hypoglycemia and suppressed serum levels of insulin and C-peptide upon endocrinological evaluation (Table 1). Contrast-enhanced computed tomography (CT) and magnetic resonance imaging (MRI) revealed a giant hypervascular tumor occupying most of the pelvic cavity, displacing and compressing the rectum. The tumor showed marked enhancement with a heterogeneous pattern suggestive of a mesenchymal tumor (Fig. 1a, b). The inferior mesenteric artery and vein were speculated to be the feeding artery and draining vein of the tumor, respectively (Fig. 1c). Colonoscopy showed no sign of tumor invasion to the mucosa of the rectum. Based on the suppressed serum levels of insulin and C-peptide accompanying the giant pelvic mesenchymal tumor, a preoperative diagnosis of SFT with NICTH (Doege-Potter syndrome) was made. During the diagnostic work-up, intravenous hyperalimentation in addition to oral normal diet was needed to control hypoglycemia; nevertheless, she often suffered from hypoglycemia, and oral or intravenous glucose intake was needed.

To prevent excessive surgical hemorrhage, preoperative TAE was scheduled 2 days before surgery. Angiography revealed that the tumor was supplied by branches arising from both the inferior mesenteric artery and the bilateral internal iliac artery. Super-selective catheterization and embolization of these vessels were performed using coil and gel-foam particles (Fig. 2). After the TAE, blood glucose level had stabilized, and no intravenous administration of glucose was needed (Fig. 3). On the other hand, the patient had fever and lower abdominal pain, which was controlled by oral analgesics. Ingestion was stopped, and preoperative
Table 1 Endocrinological evaluation

\begin{tabular}{|c|c|c|}
\hline Test & Results & Reference range \\
\hline $\begin{array}{l}\text { Plasma glucose (after overnight fast) (mg/ } \\
\quad \mathrm{dL} \text { ) }\end{array}$ & 36 & $73-109$ \\
\hline Insulin (after overnight fast) ( $\mu \mathrm{U} / \mathrm{mL})$ & $<1$ & $1.1-17.0$ \\
\hline C-peptide (after overnight fast) (ng/mL) & 0.01 & $0.8-2.5$ \\
\hline Hemoglobin A1c (\%) & 5.0 & $4.9-6.0$ \\
\hline Growth hormone (ng/mL) & 0.2 & $\leq 2.1$ \\
\hline Prolactin (ng/mL) & 20.6 & $4.1-28.9$ \\
\hline ACTH $(\mathrm{pg} / \mathrm{mL})$ & 14.9 & $7.2-63.3$ \\
\hline $\mathrm{LH}(\mathrm{mlU} / \mathrm{mL})$ & 4.9 & $0.5-68.7$ \\
\hline $\mathrm{FSH}(\mathrm{mIU} / \mathrm{L})$ & 6.7 & $1.5-168.8$ \\
\hline $\mathrm{TSH}(\mu \mathrm{IU} / \mathrm{mL})$ & 1.34 & $0.50-5.00$ \\
\hline Free T3 (pg/mL) & 2.2 & $2.3-4.0$ \\
\hline Free T4 (ng/dL) & 0.90 & $0.90-1.70$ \\
\hline Cortisol ( $\mu \mathrm{g} / \mathrm{dL})$ & 7.2 & $6.4-21.0$ \\
\hline Plasma epinephrine (ng/mL) & 0.03 & $\leq 0.1$ \\
\hline Plasma norepinephrine (ng/mL) & 0.10 & $0.1-0.5$ \\
\hline Plasma dopamine (ng/mL) & $<0.01$ & 0.82 .5 \\
\hline
\end{tabular}

Suppressed levels of insulin and C-peptide were observed, suggesting a diagnosis of non-islet cell tumor hypoglycemia

ACTH adrenocorticotropic hormone, LH luteinizing hormone, FST folliclestimulating hormone, $\mathrm{TSH}$ thyroid-stimulating hormone

mechanical bowel preparation was omitted. The fever had disappeared before surgery, and as scheduled, the tumor was safely resected with the rectum (low anterior resection with diverting ileostomy) 2 days after TAE. The operative time was $308 \mathrm{~min}$, and the estimated blood loss was $335 \mathrm{~mL}$, without the need for a blood transfusion. Considering the tumor's size and hypervascular nature, we chose open laparotomy over a laparoscopic approach.

The tumor measured $17 \mathrm{~cm}$ in diameter and weighed $850 \mathrm{~g}$. The tumor size had not reduced following embolization. The cut surface was yellowish-white in color and elastic-soft. A part of the rectum wall showed necrosis because of preoperative TAE (Fig. 4). Histologically, the tumor consisted of monotonous spindle cell proliferation without atypia. The mitosis rate was low (2/10 high-power fields). Immunohistochemical staining demonstrated that the tumor cells were positive for CD34, CD99, Bcl-2, STAT6, and vimentin, consistent with a diagnosis of SFT (Fig. 5). The tumor was also positive for IGF-II staining, and we confirmed by western blotting that the circulating high-molecular-weight IGF-II had decreased after tumor removal, concomitant with remission of hypoglycemia (Fig. 6). The patient had an uneventful postoperative course. Six months later, she underwent ileostomy closure. No evidence of recurrence has been reported in the 2-year follow-up after the initial surgery. 

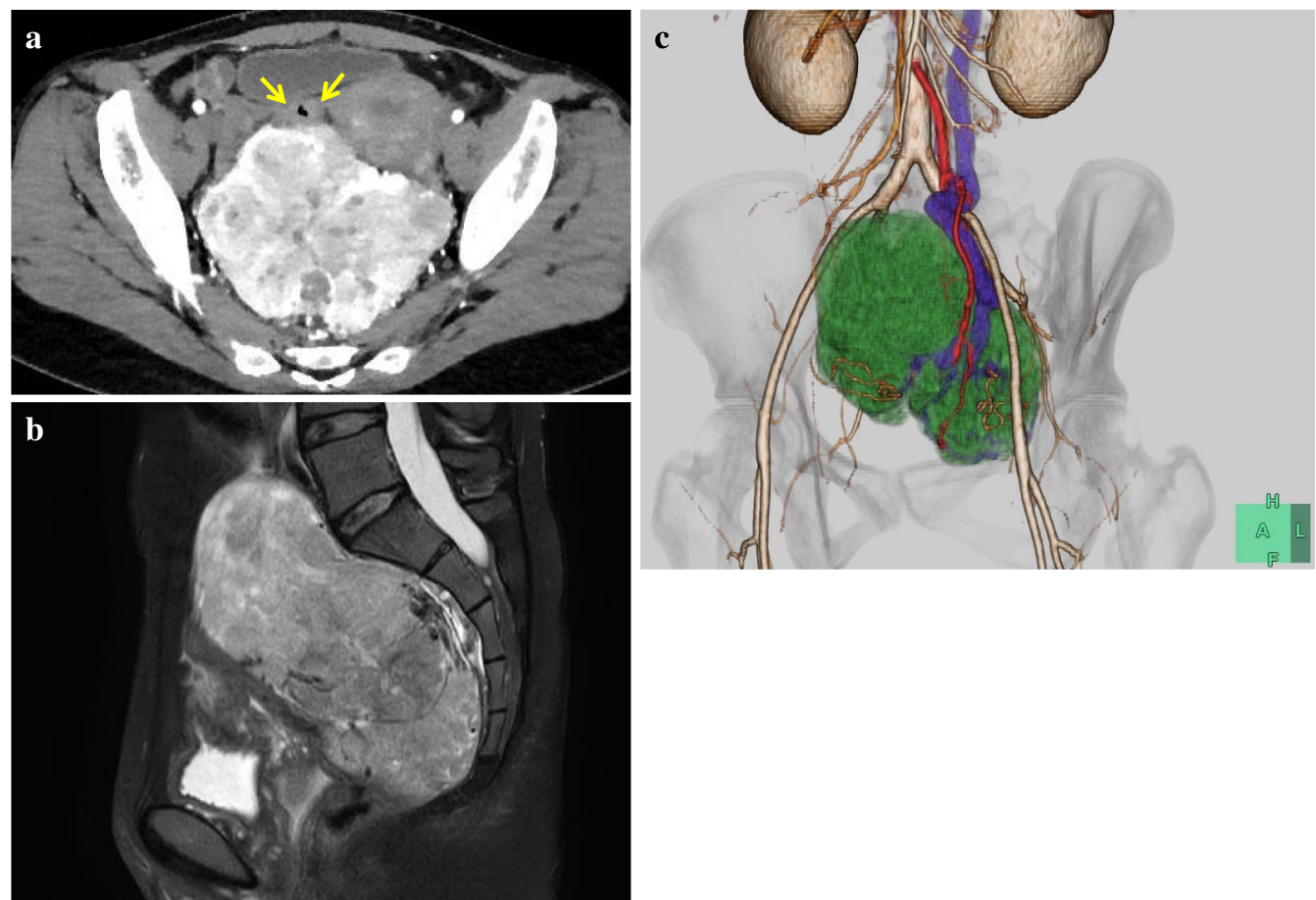

Fig. 1 Computed tomography (CT) and magnetic resonance imaging (MRI). a Contrast-enhanced CT image showed a hypervascular mass occupying the pelvic cavity, displacing and compressing the rectum (yellow arrows). The tumor showed marked enhancement in a heterogeneous pattern. b T2-weighted pelvic MRI image showed a high-signal-intensity tumor in the pelvis. c 3D-CT image reconstruction showed the tumor occupying the pelvis (green), the tumor-feeding artery (the inferior mesenteric artery colored in red), and the tumor draining vein (the inferior mesenteric vein colored in blue)

\section{Discussion}

In this case report, we present two important clinical implications. First, preoperative angiography followed by TAE enables safe and complete resection of SFTs occupying the narrow pelvic cavity, although careful management to avoid complications related to intestinal ischemia is needed. Second, embolization of the tumorfeeding artery may be an effective option in addition to medical and surgical treatment for persistent hypoglycemia due to Doege-Potter syndrome.

Although surgical resection is the definitive treatment in most cases of SFTs, large-sized tumors with a hypervascular nature that occupy the pelvic cavity frequently make surgical removal technically difficult [3]. Previous reports have revealed that surgical resection of SFTs occupying the pelvic cavity can be dangerous, associated with a large amount of intraoperative hemorrhage up to $13,660 \mathrm{~mL}$ [4-6], and may even result in intraoperative death due to uncontrollable hemorrhage [3]. Moreover, such giant tumors make it difficult for surgeons to presume their feeding arteries from preoperative imaging because of the displacement and compression of the adjacent organs by the tumor. In this context, precise preoperative identification of tumor-feeding vessels using angiography followed by TAE is a prerequisite for achieving complete resection without unexpected intraoperative hemorrhage for such giant pelvic SFTs. In our case, CT and MRI showed apparent signs of the inferior mesenteric artery as the main feeding artery of the SFT, and the involvement of the iliac artery was not suspected. However, angiography showed that the tumor was also supplied by the bilateral internal iliac artery, which led to a precise understanding of the hemodynamics of the tumor, along with safe and complete resection after TAE. Surgeons should consider preoperative angiography and TAE when planning resection of hypervascular tumors, such as SFTs, especially large tumors in the pelvic cavity, in order to achieve satisfactory results.

When performing TAE for SFTs, we should be aware of complications related to ischemia. Some authors have described the use of preoperative TAE with no complications in abdominopelvic SFTs irrigated by the iliac artery $[7,8]$. In our patient, the tumor was also supplied from the bilateral iliac artery, but the dominant vascularity was the inferior mesenteric artery. Since we were concerned about intestinal ischemia after embolization, we scheduled TAE 2 days prior to the surgery. Despite performing super-selective arterial embolization of the tumor with 

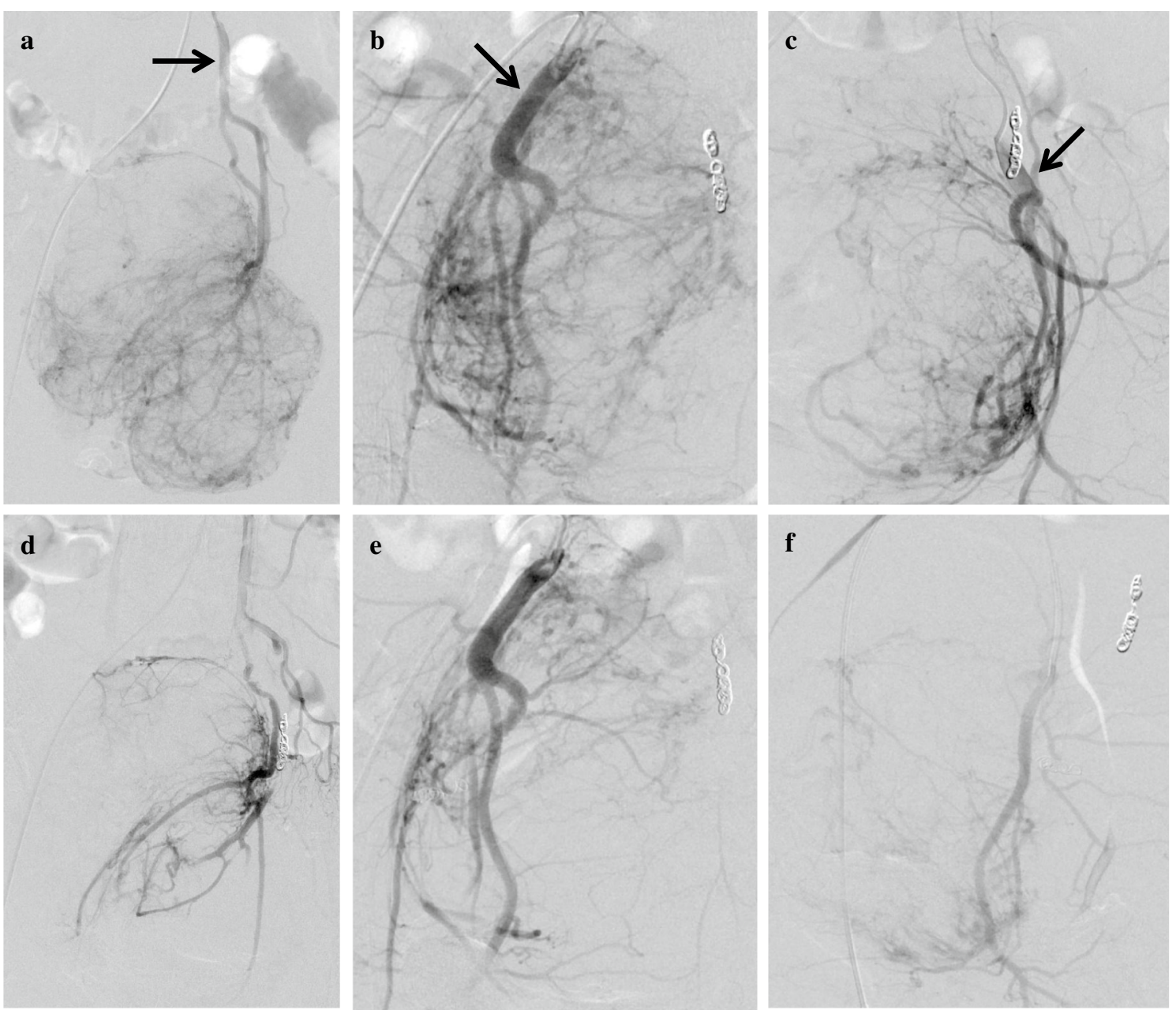

Fig. 2 Preoperative angiography and transcatheter arterial embolization. Angiography revealed that the tumor was mainly supplied by the peripheral branches of the inferior mesenteric artery ( $\mathbf{a}$, arrow) and both right (b, arrow) and left internal iliac artery (c, arrow). Super-selective catheterization and embolization of these vessels were performed using coil and gel-foam particles resulting in reduced tumor staining (d-f)

meticulous attention not to embolize the rectum and the sigmoid colon, the patient suffered from abdominal pain and fever, and operative findings revealed partial necrosis of the rectum. There are no guidelines that reveal when to perform preoperative TAE to reduce the amount of intraoperative hemorrhage. Considering the potential of intestinal ischemia, especially for tumors in which the inferior mesenteric artery is the main feeder, it is reasonable to perform such TAE within 1 or 2 days before surgery, and the patient should be carefully observed after TAE to avoid overlooking the symptoms of ischemic complications.

NICTH is most commonly described with tumors of mesenchymal or hepatic origin. Of the $288 \mathrm{NICTH}$ cases reviewed, $22 \%$ were SFTs that were commonly located in the pleura, retroperitoneum, abdomen, and pelvis [9]. Initial management of hypoglycemia in
NICTH involves increased caloric intake and frequent intravenous administration of glucose or dextrose. Total resection of the tumor is curative for hypoglycemia in many cases. For cases of uncontrollable hypoglycemia or unresectable tumors, glucocorticoid administration and local antitumor therapy, such as systemic chemotherapy [10], molecular targeted therapy [11], and radiation therapy [12] have been reported to be successful in resolving hypoglycemia. TAE for tumors was effective in treating NICTH in a hepatic fibrosarcoma case [13] but not in four cases of SFT [14]. To the best of our knowledge, this is the first case that showed TAE for a tumor to be an effective method for controlling hypoglycemia in Doege-Potter syndrome. For DoegePotter syndrome with refractory hypoglycemia or inoperable tumors, TAE may be an option for resolving hypoglycemia. 


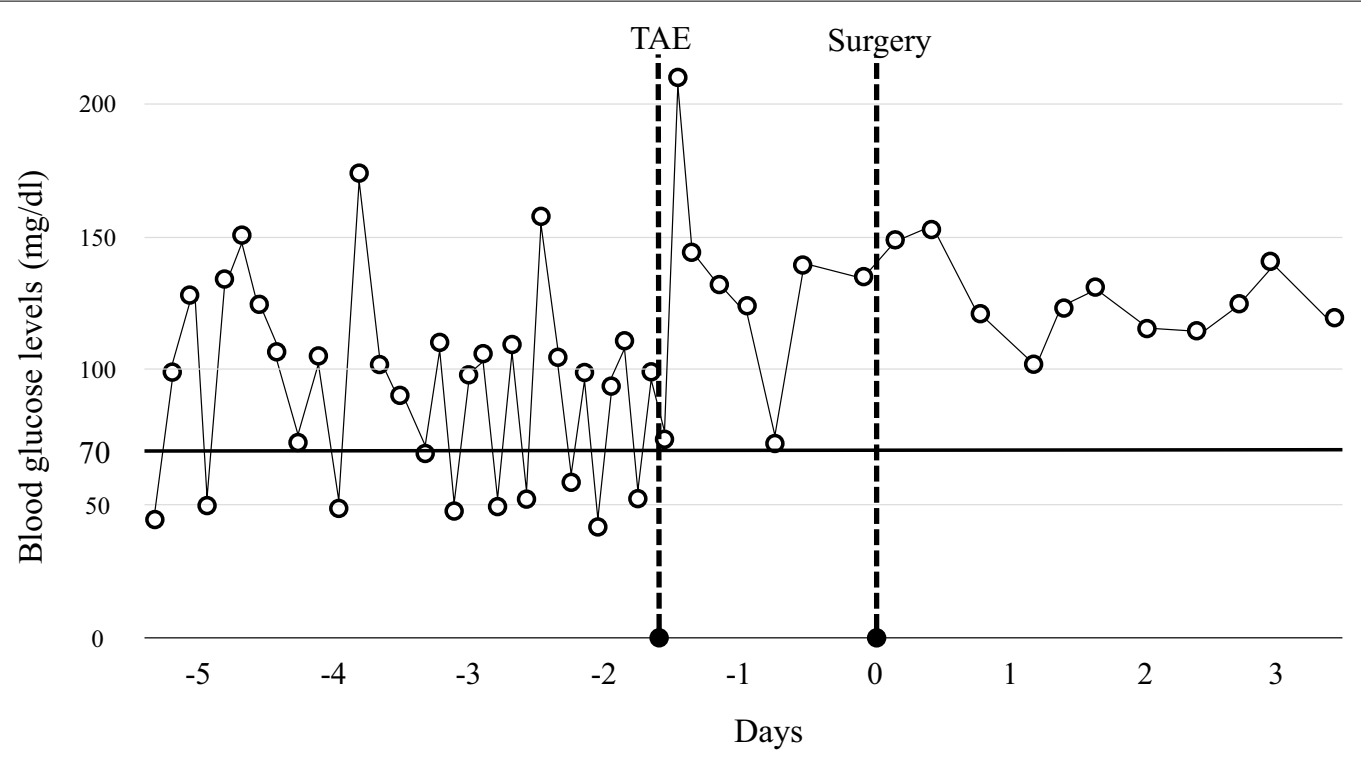

Fig. 3 Perioperative blood glucose levels of the patient. The patient frequently suffered from hypoglycemia with blood glucose level measuring under $70 \mathrm{mg} / \mathrm{dL}$ even with hyperalimentation in addition to normal ingestion, and oral or intravenous glucose intake was needed. However, after the transcatheter arterial embolization (TAE) and surgery, she did not have hypoglycemia, and the blood glucose level stabilized
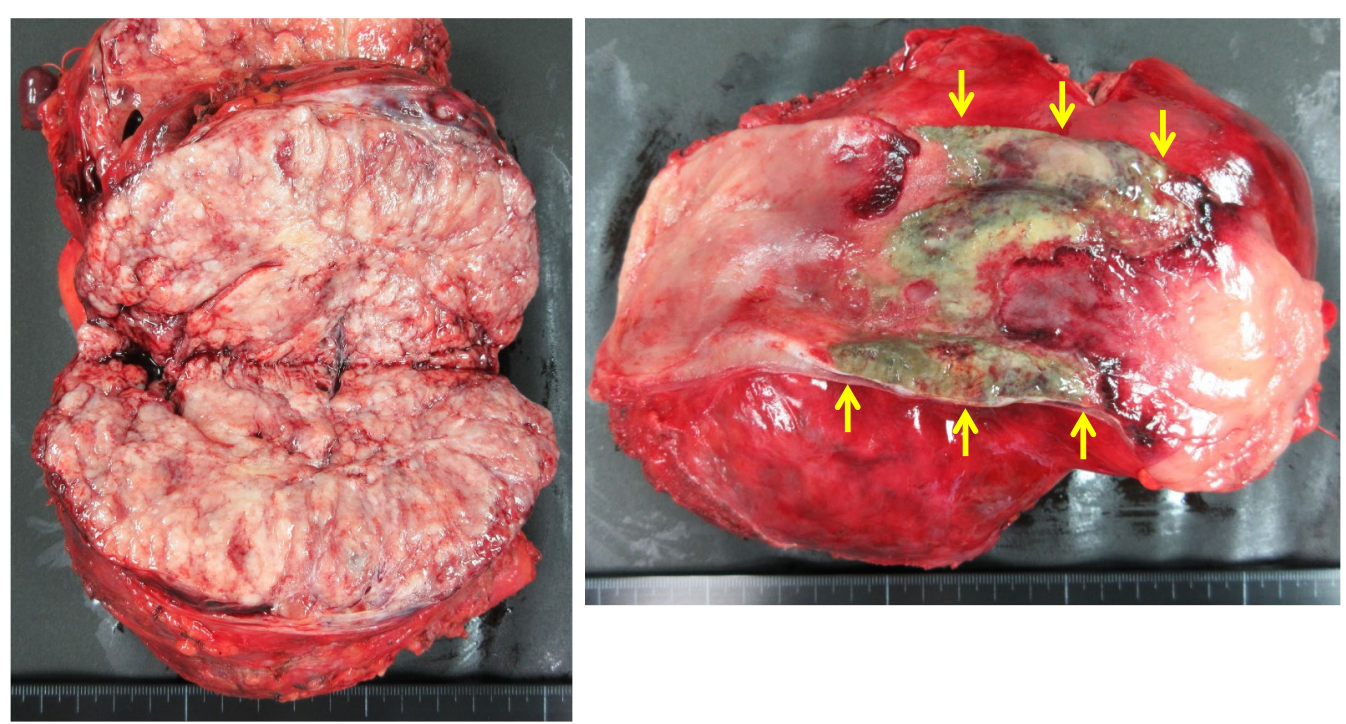

Fig. 4 Macroscopic appearance of the tumor. The cut surface was yellowish-white in color and elastic-soft. Partial necrosis of the rectum was evident as a result of preoperative transcatheter arterial embolization (yellow arrows)

\section{Conclusions}

Preoperative angiography followed by TAE enables safe and complete resection of SFTs occupying the pelvic cavity, although careful management to avoid complications related to ischemia is needed. TAE for tumors may be an option in addition to medical and surgical treatment for persistent hypoglycemia in Doege-Potter syndrome. 


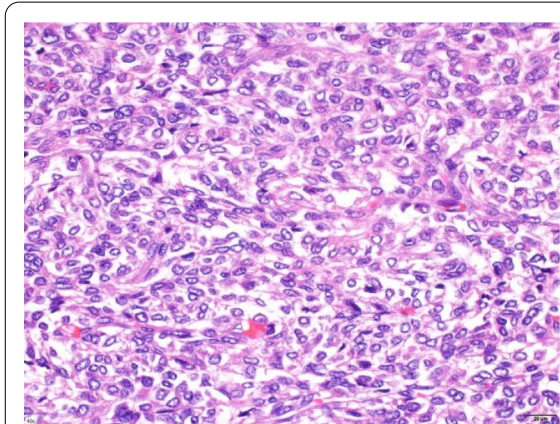

HE

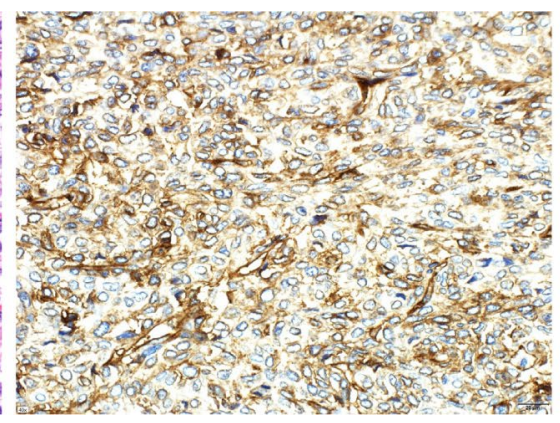

CD34

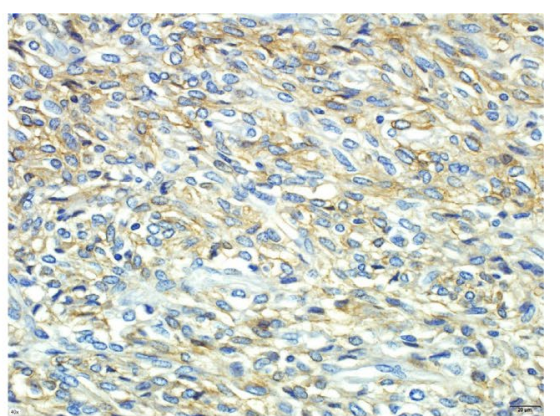

CD99

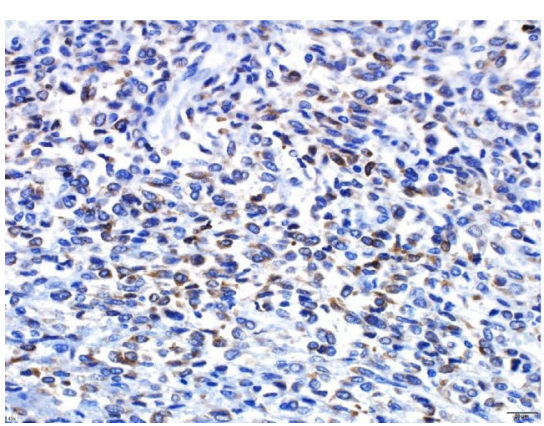

Bcl-2

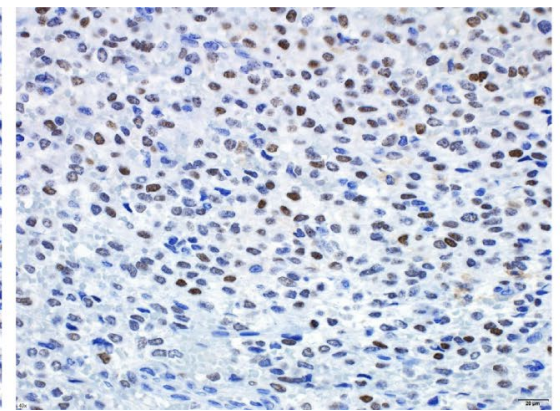

Stat6

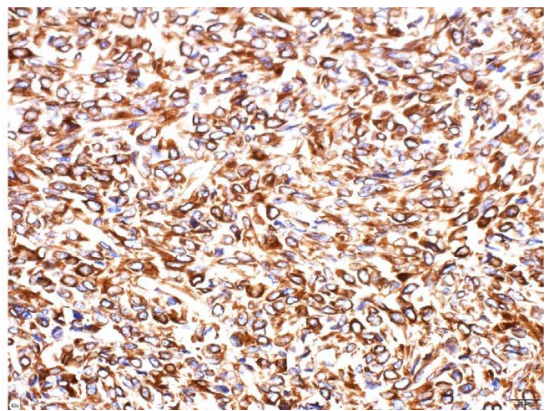

Vimentin

Fig. 5 Pathological findings. Hematoxylin and eosin staining of the tumor showed monotonous spindle cell proliferation without atypia. The mitosis rate was low (2/10 high-power fields). Immunohistochemical staining demonstrated that the tumor cells were positive for CD34, CD99, $\mathrm{BCl}-2$, STAT6, and vimentin, consistent with a diagnosis of solitary fibrous tumor

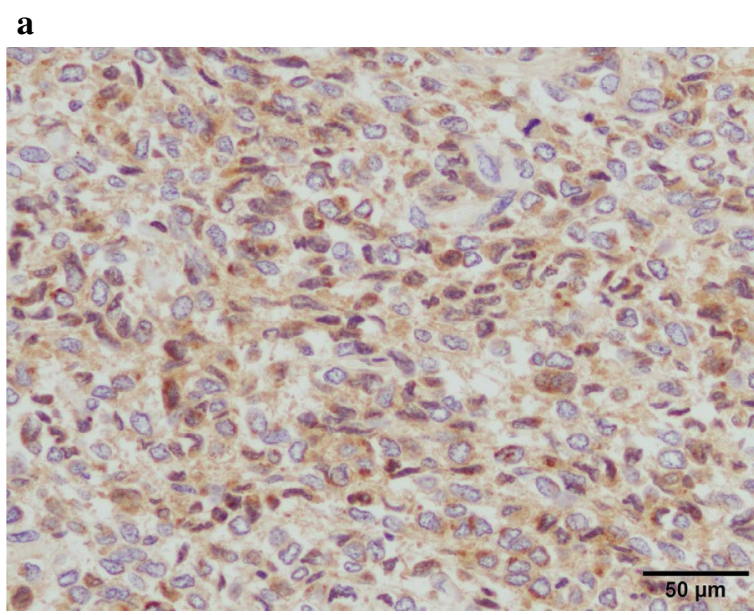

\section{IGF-II}

b

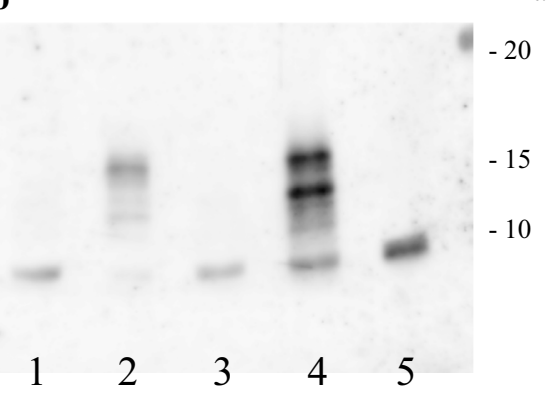

Fig. 6 Immunohistochemical staining of insulin-like growth factor-II (IGF-II) and western blotting of high-molecular-weight IGF-II. a The tumor cells were positive for IGF-II staining. $\mathbf{b}$ Lane 1, serum collected from the patient on postoperative day 5; lane 2, serum collected from the patient preoperatively; lane 3, serum collected from a healthy control; lane 4, serum collected from a different patient diagnosed as IGF-II dependent non-islet cell tumor induced hypoglycemia as a positive control of high-molecular-weight IGF-II; lane 5, standard IGF-II (7.5 kDa). Large amounts of high-molecular-weight IGF-II (approximately $16 \mathrm{kDa}$ ) were detected in the serum collected preoperatively (lane 2) but not in serum collected postoperatively (lane 1) 


\section{Abbreviations}

NICTH: Non-islet cell tumor hypoglycemia; IGF-II: Insulin-like growth factor-II; SFT: Solitary fibrous tumor; TAE: Transcatheter arterial embolization; CT: Computed tomography; MRI: Magnetic resonance imaging.

\section{Acknowledgements}

The authors are grateful to all those at the Niigata University Hospital who contributed to the care of the patients, especially the staff in the Department of Hematology, Endocrinology and Metabolism, and Department of Radiology and Radiation Oncology. We thank Y. Nagashima (Department of Surgical Pathology, Tokyo Women's Medical University, Tokyo, Japan) for the IGF-II immunohistochemistry and I. Fukuda (Department of Endocrinology, Diabetes and Metabolism, Graduate School of Medicine, Nippon Medical School) for the western blotting of IGF-II. We would like to thank Editage (www.edita ge.com) for English language editing.

\section{Authors' contributions}

$K Y$, JS, and KM are the surgeons who operated on the patient. The manuscript was drafted by KY. JS supervised the preparation of this case report. All authors read and approved the final manuscript.

\section{Funding}

No funding to declare.

\section{Availability of data and materials}

Data sharing is not applicable to this article as no datasets were generated or analyzed during the current study.

\section{Ethics approval and consent to participate}

This study was reviewed and approved by the Ethics Committee of Niigata University.

\section{Consent for publication}

Written informed consent was obtained from the patient for publication of this case report and accompanying images. A copy of the written consent is available for review.

\section{Competing interests}

The authors declare that they have no competing interests.

\section{Author details}

${ }^{1}$ Division of Digestive and General Surgery, Niigata University Graduate School of Medical and Dental Sciences, 1-757 Asahimachi-dori, Chuo-ku, Niigata, Niigata 951-8510, Japan. ${ }^{2}$ Department of Digestive and General Surgery, Uonuma Institute of Community Medicine, Niigata University Medical and Dental Hospital, Niigata, Japan.

Received: 15 August 2020 Accepted: 18 November 2020 Published online: 25 November 2020
References

1. Davanzo B, Emerson RE, Lisy M, Koniaris LG, Kays JK. Solitary fibrous tumor. Transl Gastroenterol Hepatol. 2018:3:94.

2. Fukuda I, Asai A, Nagamine T, Harada T, Tanimura-Inagaki K, Hizuka N, et al. Levels of glucose-regulatory hormones in patients with non-islet cell tumor hypoglycemia: including a review of the literature. Endocr J. 2017;64(7):719-26.

3. Wignall OJ, Moskovic EC, Thway K, Thomas JM. Solitary fibrous tumors of the soft tissues: review of the imaging and clinical features with histopathologic correlation. AJR Am J Roentgenol. 2010;195(1):W55-62.

4. Kim MY, Jeon S, Choi SD, Nam KH, Sunwoo JG, Lee JH. A case of solitary fibrous tumor in the pelvis presenting massive hemorrhage during surgery. Obstet Gynecol Sci. 2015;58(1):73-6.

5. Wada Y, Okano K, Ando Y, Uemura J, Suto H, Asano E, et al. A solitary fibrous tumor in the pelvic cavity of a patient with Doege-Potter syndrome: a case report. Surg Case Rep. 2019;5(1):60.

6. Soda H, Kainuma O, Yamamoto H, Nagata M, Takiguchi N, Ikeda A, et al. Giant intrapelvic solitary fibrous tumor arising from mesorectum. Clin J Gastroenterol. 2010;3(3):136-9.

7. Yokoyama Y, Hata K, Kanazawa T, Yamaguchi H, Ishihara S, Sunami E, et al. Giant solitary fibrous tumor of the pelvis successfully treated with preoperative embolization and surgical resection: a case report. World J Surg Oncol. 2015;13:164.

8. Zeron-Medina J, Rodriguez-Covarrubias F, Garcia-Mora A, GuerreroHernandez M, Chable-Montero F, Albores-Saavedra J, et al. Solitary fibrous tumor of the pelvis treated with preoperative embolization and pelvic exenteration. Am Surg. 2011:77(1):112-3.

9. Bodnar TW, Acevedo MJ, Pietropaolo M. Management of non-isletcell tumor hypoglycemia: a clinical review. J Clin Endocrinol Metab. 2014;99(3):713-22.

10. Huang JS, Chang PH. Refractory hypoglycemia controlled by systemic chemotherapy with advanced hepatocellular carcinoma: a case report. Oncol Lett. 2016;11(1):898-900.

11. Singhal A, Hadi R, Mehrotra K, Rastogi S, Masood S. Paraneoplastic hypoglycaemia: a rare manifestation of pelvic gastrointestinal stromal tumour J Clin Diagn Res JCDR. 2017;11(2):Xd01-Xd2.

12. Kishi K, Sonomura T, Sato M. Radiotherapy for hypoglycaemia associated with large leiomyosarcomas. Br J Radiol. 1997:70:306-8.

13. Kotani K, Tsuji M, Oki A, Kashihara T, Yamada K, Kawakami F, et al. IGF-II producing hepatic fibrosarcoma associated with hypoglycemia. Intern Med. 1993;32(12):897-901.

14. Han G, Zhang Z, Shen X, Wang K, Zhao Y, He J, et al. Doege-Potter syndrome: a review of the literature including a new case report. Medicine. 2017:96(27):e7417.

\section{Publisher's Note}

Springer Nature remains neutral with regard to jurisdictional claims in published maps and institutional affiliations.

\section{Submit your manuscript to a SpringerOpen ${ }^{\circ}$ journal and benefit from:}

- Convenient online submission

- Rigorous peer review

- Open access: articles freely available online

- High visibility within the field

Retaining the copyright to your article

Submit your next manuscript at springeropen.com 